\title{
Meaning In Life as a Moderator of Stress in Undergraduate Students
}

\author{
Yousaf Raza $^{1^{*}}$, Amna Noureen ${ }^{2}$, Zarnab G. Bhatti ${ }^{3}$ and Haziq Mehmood ${ }^{4}$ \\ ${ }^{1}$ Fellow of College of Physicians and Surgeons, Pakistan, MSc Psychology, Academic Associate logotherapy. \\ ${ }^{2}$ MS Clinical Psychology, Institute of Psychiatry, Benazir Bhutto Hospital, Rawalpindi, Pakistan \\ ${ }^{3}$ MBBS, Rawalpindi Medical University, Rawalpindi, Pakistan. \\ ${ }^{4} \mathrm{PhD}$, University of Lingnan, Hong Kong.
}

*Corresponding Author: Yousaf Raza, Fellow of College of Physicians and Surgeons, Psychology, Academic Associate logotherapy, Islamabad, Pakistan. E-mail: usufraza@gmail.com

Received Date: November 30, 2019; Accepted Date: December 20, 2019; Published Date; December $24,2019$.

Citation: Yousaf Raza, Amna Noureen, Zarnab G. Bhatti and Haziq Mehmood. Meaning In Life as a Moderator of Stress in

Undergraduate Students, J. Psychology and Mental Health Care. Doi: 10.31579/2637-8892/062.

Copyright: ( 2019 Yousaf Raza, This is an open-access article distributed under the terms of the Creative Commons Attribution License, which permits unrestricted use, distribution, and reproduction in any medium,provided the original author and source are credited.

Abstract
Stress is a common predisposing, precipitating, and perpetuating factor of psychological and physical illnesses. Various
studies have shown a beneficial role of having a meaning in life with regards to stress. There are no local studies available
that have studied the correlation between the two constructs of perceived stress and meaning in life, in the Pakistani
population. Therefore this study aims to determine correlation between scores of MIL scale and scores of Stress, in
undergraduate students.
A total of 254 undergraduate students were selected as respondents in the study. They were assessed for perceived stress
and meaning in life using Perceived Stress Scale $(\mathrm{PSS})$ and the Purpose in Life Scale (PIL) respectively. It was a cross
sectional study, demographic and clinical variables were collected via a Performa.
On average females scored higher $(\mathrm{M}=2.20, \mathrm{SD}=0.70)$ than males $(\mathrm{M}=1.78, \mathrm{SD}=0.52$,) on perceived stress. While
males scored $(\mathrm{M}=3.57, \mathrm{SD}=0.55)$ significantly $\mathrm{t}(244)=4.69, \mathrm{p}<.05)$ higher on purpose in life questionnaire as
compared to females $(\mathrm{M}=3.21, \mathrm{SD}=0.69)$. Correlation coefficient was found to -0.52 representing a significant negative
correlation between the two variables. $\mathrm{R}^{2}$ came out to be 0.27 signifying that $27 \%$ of the variance in perceived stress is
accounted by meaning in life.
There is a strong correlation between meaning in life and perceived stress. Further it was also found that female gender
was more vulnerable to stress and less likely to score high on meaning in life. Conversely male gender was more likely to
score higher on meaning in life and lower on perceived stress.
Keyword: moderator stress; psychology; physical illness

\section{Introduction}

Stress, according to Hans Selye, has received the mixed blessing of being known too well but understood too little. Although no exact consensus exists in defining the term, it is usually understood as the brain's response to perceived or real demands and/or challenges (stressors) (Sadock, et al., 2015). Stress is universally recognized as a pre-disposing, precipitating, and perpetuating factor for almost all psychiatric illnesses. The most elaborately studied and explicated of all mental illnesses is depression. Yanga, et al., (2015) in a review article explicated the multi-tiered relationship between stress and depressive illness. They enlist the prefrontal cortex, the ventral tegmental area, and the hippocampus as the main brain regions involved through which stress transpires depression.

Several studies (McAndrew \& Warne, 2014; Bisagno, \& Cadet, 2014; Yau \& Potenza, 2013; Theoharides et al., 2012) have related stress with self harm, addictive behaviors, abnormal eating disorders, PTSD and Autism spectrum disorder. In the context of bipolar affective disorder, unsurprisingly, stress plays a vital role particularly in high-risk individuals with a biological predilection. Younger children of a bipolar parent when exposed to such stressors may exhibit developmental delays, anxiety, or sleep disturbances, whereas adolescents may manifest adjustment and minor mood disorders. In adulthood they may experience recurrent depressive episodes before going on to develop bipolar disorder (Duffy et al., 2016). Even in the realm of psychotic disorders in adults stress has been identified as a major player. According to a meta-analysis genetic vulnerability, compounded by early childhood adverse experiences, render the individual more susceptible to developing schizophrenia as a consequence of stressors in adulthood (the 3-Hit hypothesis) (Daskalakis \& Binder, 2015)

Stress, perhaps more than any other phenomenon, reflects the dynamic relationship between the psychological and somatic dimensions of human existence. In an extensive review Purdy discussed the stress as a risk factor for multiple chronic illnesses including cardiovascular disease, metabolic syndrome, autoimmune diseases, chronic pain and gastrointestinal diseases (Purdy, 2013). In addition stress has been empirically shown to lead to decreased immunity, increased incidence of infections, and a plethora of other diseases affecting all systems of the body (Sadock et al., 2015).

Psychological importance of MIL was systematized by Viktor E. Frankl in the form of logotherapy, the third Viennese school of psychotherapy. Frankl proposed that pursuit of a MIL is the primary motivation of human beings and is therefore protective with regards to mental health, 
contributes positively towards psychological well-being, and is thus preventive against various psychiatric disorders. Wherever pain is inevitable due to biological or psychological illnesses, a meaningful perspective alleviates the suffering by making it more understandable and thereby tolerable (Frankl, 2014).

In the context of responding to stressful life events Crystal Park suggests a framework of meaning-making. She said that meaning in life is a core human motive derived from human need for significance, comprehension, and transcendence. Specifically the meaning-related needs include agency, control, certainty, identity, social validation, values, and mortality defense. A well-functioning meaning system results in an experience of peace and satisfaction, she contends. A poor functioning system manifests as significant distress. A positive sense of life meaning results when individuals feel they comprehend the world, their place therein, and are making adequate progress towards their goals consistent with their values (Park, 2010; Park, 2017).

Paul Halama showed that people with low levels of meaningfulness in their lives demonstrated emotion-based, avoidant, and maladaptive coping methods comprising of drug abuse, denial, and mental or behavioral disengagement as compared to people who had high levels of meaningfulness who exhibited adaptive coping (Halama, 2014). Several studies showed relationship between Meaning in life and positive psycology. In an analysis of 1,997 adolescent students in Romania, Brassai et al (2011) concluded that meaning in life has a positive impact on quality of life and psychological well-being while it decreased the incidence of drug use and sedentary life-style. Steger and Kashdan (2013) in a study showed that those with greater instability of daily meaning were found to have lower daily levels of meaning in life, and lower global levels of life satisfaction, positive affect, social connectedness and relationship satisfaction. Damasio et al (2015) showed that in those categorized under the "meaningfulness" subgroup fared better in multiple domains including self-knowledge, generativity, harmony, freedom, community, and love. Krok (2015) in a study of 187 participants showed that a presence of meaning correlated positively and significantly with psychological well-being $(0.47, \mathrm{p}<0.001)$ and in a second study of 182 participants, published in the same paper, that personal meaning correlated with psychological well-being $(0.54, \mathrm{p}<0.001)$. Further, Hill and Turiano (2014) in a longitudinal study of 7,108 individuals of whom 569 died, that those dying had scored significantly low on the purpose in life scale $(\mathrm{t}=10.65 ; \mathrm{p}<0.05)$. They concluded thereby that purpose predicts longevity.

Strong relationship has been established between meaning in life and illnesses. Steger et al. (2009) concluded in a cross-sectional survey that there is a negative correlation between presence of meaning and depressive illness $(\mathrm{r}=-0.60, \mathrm{P}<0.001)$, and anxiety $(\mathrm{r}=-0.43, \mathrm{P}<$ $0.001)$, and a positive correlation with perceived health $(\mathrm{r}=0.36, \mathrm{P}<$ 0.001). Martin et al (2011) cited that a high sense of meaning in life decreases the possibility of using substances of dependence. Sense of meaning serves as a coping resource that is a preventive factor in highrisk situations. Conversely a low sense of meaning in life is evidenced as both a cause and effect of dependent alcohol use. In an interventional study Martin et al concluded that a lower sense of meaning in life significantly predicted use of cocaine $(\mathrm{B}=-.04, \mathrm{SE}=.016, \mathrm{OR}=.96,<$ $.05)$ and use of alcohol $(\mathrm{B}=-.05, \mathrm{SE}=.015, \mathrm{OR}=.96, \mathrm{p}<.05)$. Similarly low meaning in life increased the chances of relapse with a $\chi^{2}(101)=$ $135.69, \mathrm{p}<.01$, for cocaine relapse, and $\chi 2(101)=133.54, \mathrm{p}<.01$, for alcohol relapse. Marco et al (2017) in an analysis of 124 women with borderline personality disorder concluded that meaning in life negatively moderated the association between suicide risk factors and hopelessness. Wilchek-Aviad and Malka (2016) concluded after a study of 450 Jewish adolescents that meaning in life correlated significantly and negatively with suicidal tendencies.

Literature search for MIL related research in Pakistan yielded scanty results. There was no study on this subject published in any indexed journal. It is to compensate for this dearth of research in Pakistan that this study is proposed. This study will set the pace for further research in this area. If the results in this study are consistent with those in international studies given above, and having a Meaning in Life is discovered to moderate perceived stress levels in the Pakistani population, then that will pave the way for researching psychotherapies oriented towards guiding patients towards meaning discovery in their lives. Furthermore interventional studies looking to employ meaning-centered psychotherapies in treatment of psychiatric illnesses may be designed.

Further, increasing awareness in general public of the importance of MIL in reducing perceived stress, and thereby preventing psychiatric illnesses, will strongly contribute towards the domain of preventive psychiatry. Since stress is a perpetuating factor in psychiatric and physical illnesses, MIL awareness will help attenuate the severity of these illnesses and decrease rates of relapses in recurrent disorders. This will help to keep pace with research and interventions in this area around the world. Since there is no parent study to draw data for calculation of minimum-required sample size, a pilot study of 30 participants was carried out. The correlation coefficient rho came out to be -0.648 .

\section{Methods}

This descriptive cross-sectional study was conducted on undergraduate medical students. Since there is no parent study to draw data for calculation of minimum-required sample size, a pilot study of 30 participants was carried out. The correlation coefficient rho came out to be -0.648 .With the help of Statstodo Sample Size Calculator, keeping Level of significance $=5 \%$, Confidence Interval $=95 \%$, Correlation coefficient $\mathrm{rho}=-0.648$ (calculated from the pilot study), the minimally required sample size came out to a mere 21 , however we arbitrarily chose to obtain the sample of over 250 students. The sample was obtained in November 2016. Sampling technique was non-probability consecutive sampling. Our inclusion criterion was all undergraduate medical students between the ages of 18 and 26 years. Our exclusion criteria was anyone on treatment for a diagnosed psychiatric illness.

After obtaining approval from the Hospital Ethics Committee, students were sampled. The purpose of the study was explained to each participant and written informed consent was obtained. A structured questionnaire containing demographic details (age, gender, year of study, place of residence, boarding status), Perceived Stress Scale (PSS), and Purpose in Life Test (PIL) were then administered.

All the data was entered and analyzed in Statistical Package of Social Sciences, SPSS (version 22). The quantitative variables of the study like age, score on PSS, and score on PIL were calculated as mean and standard deviation. The qualitative variables like gender, year of study, and whether participant is a hostelite or day-scholar were calculated as frequencies and percentages.

To determine any existing correlation, Pearson's correlation test was applied at 5\% level of significance and Pearson product-moment correlation coefficient was calculated along with $p$ value $(\leq 0.05$ taken as significant). Effect modifiers like age, gender, year of graduation, socioeconomic status, residence, were controlled by hierarchical multiple regression; with perceived stress, study year, age, boarding status and marital status as predictors of meaning in life among medical students.

\section{Results}

\section{Sample demographics}

The study was conducted from November, 2016 to April 2017 and the total number of study participants was 254 . There were a total of $65.65 \%$ females and $34.25 \%$ males in the sample. We obtained information regarding the proportion of males and females in population which was $35 \%$ and $65 \%$ respectively. As proportion of males and females in sample $(34.25 \%$ and $65.65 \%$ ) is approximately equal to the 
proportion in population, the need to give post stratification weights to the cases across gender was precluded. In the sample, $57.48 \%$ students were boarders while $42.52 \%$ were non-boarders. Majority $(94.9 \%)$ of the participants were unmarried, while $5.1 \%$ were married.

Most of the respondents in our study were studying the fourth year of their MBBS constituting $35.8 \%$ of the total number. Final year students constituted $25.98 \%$ and third year students made up $25.20 \%$ of the responding sample. First year $(7.09 \%)$ and second year $(5.9 \%)$ MBBS students were the least represented in the sample. The proportion of students in the five years of MBBS was $18 \%, 20 \%, 20 \%, 20 \%, 20 \%$ for study year, 1, 2, 3, 4, 5 respectively, which are different from their respective proportions in population (According to the information provided by the Department of Medical Education). We used poststratification weight to minimize this bias in our sample. We calculated weight for each category of study year using the following formula:

Weight $=$ Proportion of population/ proportion of sampl

After giving the weights to the cases we observed that the proportion of study years in population is similar to that in the data provided by Department of medical Education. (See Table no.1) We will use weighted data for further analysis here on in.

\begin{tabular}{|c|c|}
\hline Ranges & Frequencies \\
\hline $0-9$ & 9 \\
\hline $10-19$ & 101 \\
\hline $20-29$ & 117 \\
\hline $30-40$ & 27 \\
\hline Total & 254 \\
\hline
\end{tabular}

Table 1: Representing percentage and frequency of student's study years $(1,2,3,4,5)$ in sample after post stratification weight

The mean score on the Perceived Stress Scale was 20.56 (SD \pm 6.71$)$. The minimum score attained was 0 and the maximum was 39 . The MIL score on the Purpose in Life scale ranged from 20-100. The maximum score attained was 100 and the minimum was 23 , with a mean of 66.78 $(\mathrm{SD} \pm 13.49)$.

Table no. 2 shows frequencies of the scores of PIL measured across arbitrarily assigned ranges. Most respondents $(n=156)$ scored in the range of 50-74.

\begin{tabular}{|c|c|c|}
\hline Students study's year & n & \% \\
\hline First & 44 & 16.30 \\
\hline Second & 70 & 26.10 \\
& & \\
Third & 52 & 19.50 \\
\hline Fourth & 50 & 18.80 \\
\hline Fifth & 51 & 19.20 \\
\hline Total & 268 & 99.20 \\
\hline
\end{tabular}

Table 2: Ranges of raw score on purpose in life (pil) and respective frequencies

Table no. 3 shows the frequencies of the scores of PIL measured across arbitrarily assigned ranges. A total of 110 respondents ranged between 0 19, 117 respondents scored between20-29, and 27 scored between 30-40 signifying significant levels of perceived stress.

\begin{tabular}{|c|c|}
\hline Ranges & Frequencies \\
\hline $0-9$ & 9 \\
\hline $10-19$ & 101 \\
\hline $20-29$ & 117 \\
\hline $30-40$ & 27 \\
\hline Total & 254 \\
\hline
\end{tabular}

Table No. 3: Ranges of Raw Score on Perceived Stress (Ps) and Respective Frequencies

We conducted Pearson Product Moment Correlation and found a significant negative relationship between perceived stress and purpose in life $\left(\mathrm{r}=-0.57, \mathrm{R}^{2}=0.27, p<.01\right)$ is indicated that $27 \%$ of the variance in perceived stress is accounted by meaning in life or in other words perceived stress and purpose in life has $27 \%$ of shared variance. The scatterplot in figure no. 1 represents that there are no potential outliers that needed to be investigated as most points seem to fall within the vicinity of other points.

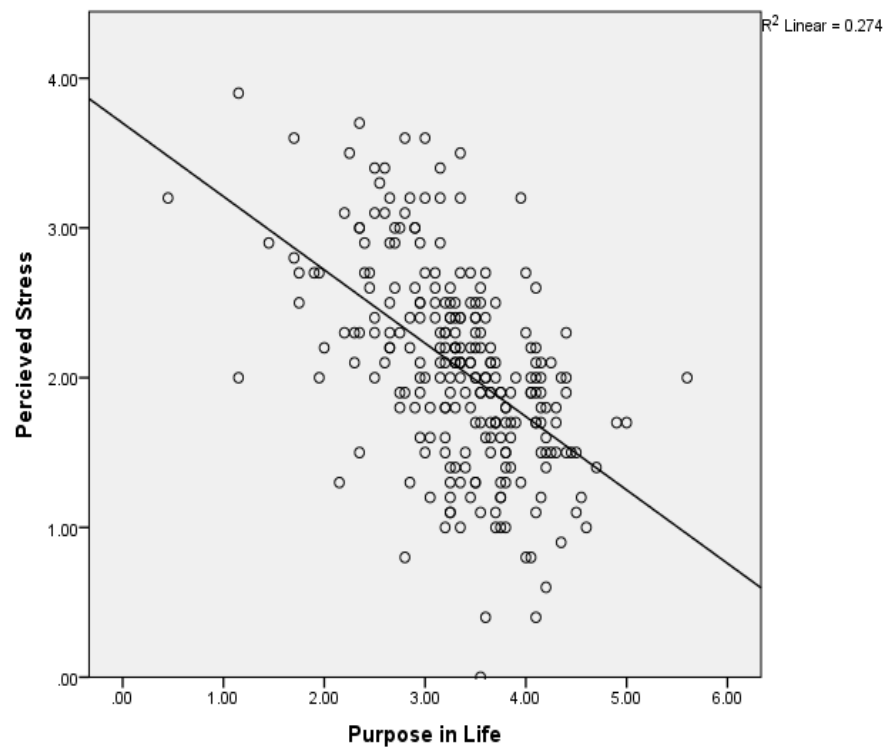

\section{Figure no. 1: Scatterplot of Purpose in Life and Perceived Stress}

We conducted hierarchical multiple regression with perceived stress, study year, age, boarding status and marital status as predictor of meaning in life among medical students. Initially we ran bivariate regression analysis by using only one predictor "perceived stress", which came out to be significant predictor of meaning in life as shown in Table no. 4 under step 1. Further on we conducted hierarchical multiple regression analysis including perceived stress, study year, gender, age, boarding status and marital status as predictors of meaning in life. The $\mathrm{R}$ squared change was not found significant and when we assess individual predictors like age, boarding status and marital status the resultant $p$ values come out to be greater than 10, which indicated that these three predictors are not significantly improving the variance in the model. We excluded these variables and then conducted analysis by remaining predictors which were perceived stress, study year and gender as shown in Table no. 4 under step 2. Perceived stress found to be significant predictor of meaning in life, the negative sign associated with beta value of stress indicated that as the stress scores go up the meaning in life goes down and vice versa. This relationship between perceived stress can also be interpreted in the way that perceived stress has a negative relationship with meaning in life among medical students after controlling for gender and study year. In other words, the greater the meaning in life of an individual the lesser the perceived stress is going to be. The variance accounted by perceived stress on meaning in life cannot be attributed to the gender and study year but 
this relationship exists regardless of gender or study year to which students belong.

Study year is a significant predictor of meaning in life after controlling for stress and gender. This relationship is surprising that more senior students have less meaning in life, as higher years are associated with low meaning in life. Gender has a marginally significant negative association with meaning in life after controlling for stress and year of study. Males have scored significantly more on meaning in life as compared to females. Our findings indicate that perceived stress and purpose in life is not significantly related to the age of the individuals.

\begin{tabular}{|c|c|}
\hline Ranges & Frequencies \\
\hline $0-9$ & 9 \\
\hline $10-19$ & 101 \\
\hline $20-29$ & 117 \\
\hline $30-40$ & 27 \\
\hline Total & 254 \\
\hline
\end{tabular}

\section{Table 4}

Hierarchical Multiple Regression with Perceived Stress (ps), Study Year and Age as Predictors of Meaning in life

Note. $R^{2}=.268$ for Step $1, R^{2}$ Change $=.031 * *$ for Step $2(p<.01), * p<$ .10 (marginally significant) ${ }^{* *} p<.01, * * * p<.001, B$ Unstandardized Regression Coefficient, SE B Standard Error associated with B, $\beta$ Standardized Regression coefficient

\begin{tabular}{|c|c|c|c|}
\hline & $B$ & $S E B$ & $\beta$ \\
\hline Step 1 & & & \\
\hline Constant & 3.78 & .20 & $-.52^{* * *}$ \\
\hline PIL & -.52 & .06 & \\
\hline Step 2 & & & $-.48^{* * *}$ \\
\hline Constant & 3.32 & .32 & -.63 \\
\hline PIL & -.48 & .06 & $-.18^{* *}$ \\
\hline Study Year & -.02 & .04 & \\
\hline Gender & -.25 & .08 & \\
& & & \\
& & & \\
\hline
\end{tabular}

Figure no. 1: Scatterplot of Purpose in Life and Perceived Stress

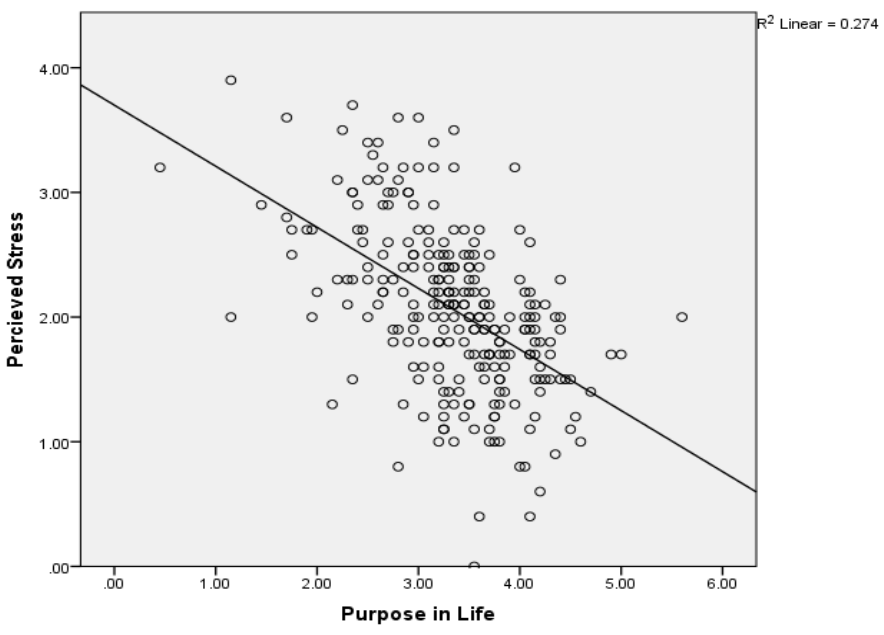

Auctores Publishing-Volume 3(5)-10062 www.auctoresonline.org Page-4

\section{Discussion}

The aim of our study was to assess the influence of meaning in life in on psychological stress. Undergraduate students at the college were enrolled for the study. A rigorous statistical analysis of results went on to substantiate our hypothesis. It was found that there is a negative correlation between perceived stress and meaning in life. This correlation was statistically significant and persisted despite controlling for different variables. Female respondents scored higher on the perceived stress scale and male respondents fared better on the purpose in life scale. Additionally, more senior students were found to have lesser levels of meaning in life than junior students.

Our study was unique in the local context as similar researches on the subject could not be discovered in our population. Additionally, the more recent literature in the international studies pertained to more complex constructs of meaning in life with robust and advanced study designs; ours was relatively basic in comparison. For these reasons drawing comparisons there between would be difficult. However, similarities may still be inferred as is shown here on in.

Various studies claim that medical students undergo greater stress than non-medical students. Although this wasn't one of our study aims, the high rates of stress shown (the mean score on PSS was 20.55 with a SD of 13.50) underscores the importance of this area of study. Saeed et al (2016) conducted a study among medical students in a Saudi university. They concluded that $33.3 \%$ of their students were severely stressed and $30 \%$ were well. They further concluded that female gender constituted an 11.8 times risk for being more stressed. This is comparable with our results as the female part of our population was also more likely to be score higher in the PSS $(\mathrm{M}=2.20$ compared to the males where $\mathrm{M}=1.78$.

Cavallo et al (2016) in a study of university students found that the score on PSS-10 had a mean of 32.2 (SD 4.6, 95\% CL 31.6-32.7), slightly more comparable to the mean of our student sample. Their student sample also revealed a higher degree of stress of in female respondents.

Cole et al (2015) showed that Meaning in Life outplayed the effects of loneliness in predicting leukocyte gene expression profiles.

Subramaniyan et al (2014) showed that those whose meaning in life was high scored low on perceived stress in a population in Tamil Nadu. Specifically they outlined that those whose meaning corresponded to adhering to social norms, whether religious, professional, or family, scored low on stress scales $(\mathrm{X} 2=3.536, \mathrm{p}=0.04)$. Although our study did not compute the individual items on the scale, the results are nevertheless consistent and comparable. Similarly Shand et al (2015) conducted a study to see the relationship between different psychosocial factors and post-traumatic stress and posttraumatic growth in oncology patients. They concluded that posttraumatic growth was positively correlated with spirituality $(r=0.33)$ and religiosity $(r=0.36)$. Our results are in consistency with Shand et al's conclusion that meaning in life, via spirituality and religiosity, is associated with post-traumatic growth.

Mohbat-Bahar et al (2014) have already shown the efficacy of psychological interventions that promote meaning in life (logotherapy) in decreasing anxiety in patients of breast cancer. Prior to the intervention the mean anxiety scores of the test group were 39.33 with a SD of 5.74 and the control group had a mean of 32.20 with a SD of 8.06. Following the intervention the test group scored a mean of 13.93 with an SD of 5.15 whereas the control group showed a slight increase in their anxiety scores.

Saleem and Saleem (2017) in a local study recently published showed the positive role of religiosity in psychological well-being. They studied a sample of 120 medical and 120 non-medical students from universities in Islamabad. Although the construct we assessed was more general (religiosity is one source of MIL) and the correlation studied in their study 
9. Halama P. (2014). Meaning in life and coping: Sense of meaning as a buffer against stress. In Meaning in positive and existential psychology (pp. 239-250). Springer, New York, NY.

10. Hill PL and Turiano NA. (2014). Purpose in life as a predictor of mortality across adulthood. Psychological science, 25(7): 1482-1486.

Our study-design was cross-sectional. Meaning related constructs should be studied on a more longitudinal study design. We used non-probability sampling technique. A more representative populations sampling should be done in the future. Correlational studies can't be used to make causal claims. More robust and sophisticated study designs should be implied to establish causal relationships. Self-reporting questionnaires were employed and therefore subjectivity and biases in responses could not be controlled for.

More refined and standardized scales and questionnaires may be used in the future. The ones employed may have had confounding variables and did not include sub-scales to assess for sub-constructs.

\section{Conclusion}

Stress is one of the most common problems that places serious constraints on a person's life activities and predisposes them to many illnesses. This study found that this problem was significant in medical students sampled. More importantly this study shows that there is a strong correlation between meaning in life and perceived stress. Further it was also found that female gender was more vulnerable to stress and less likely to score high on meaning in life. Conversely male gender was more likely to score higher on meaning in life and lower on perceived stress.

\section{Declaration of interest}

The authors report no conflicts of interest. The authors alone are responsible for the content and the writing of the paper.

\section{References}

1. Bisagno V and Cadet JL. (2014). Stress, gender, and addiction: potential roles of CRF, oxytocin and argininevasopressin. Behavioural pharmacology, $25\left(\begin{array}{lll}5 & 0 & 6\end{array}\right)$ : 445.

2. Brassai L, Piko BF and Steger MF. (2011). Meaning in life: Is it a protective factor for adolescents' psychological health? International journal of behavioral medicine, 18(1): 44-51.

3. Cavallo P, Carpinelli L and Savarese G. (2016). Perceived stress and bruxism in university students. BMC research notes, 9(1): 514.

4. Cole SW, Levine ME, Arevalo JM, Ma J, Weir DR and Crimmins EM. (2015). Loneliness, eudaimonia, and the human conserved transcriptional response to adversity. Psychoneuroendocrinology, 62: 11-17.

5. Damásio BF and Koller SH. (2015). Complex experiences of meaning in life: Individual differences among sociodemographic variables, sources of meaning and psychological functioning. Social Indicators Research, 123(1): 161-181.

6. Daskalakis NP and Binder EB. (2015). Schizophrenia in the Spectrum of Gene-Stress Interactions: The FKBP5 Example. Schizophrenia bulletin, 41(2): 323-329.

7. Duffy A, Jones S, Goodday S and Bentall R. (2016). Candidate risks indicators for bipolar disorder: early intervention opportunities in high-risk youth. International Journal of Neuropsychopharmacology, 1-10. Epub ahead of print 25 June 2015. DOI: 10.1093/ijnp/pyv071

8. Frankl VE. (2014). The Existential Vacuum: A Challenge to Psychiatry. In: Will to Meaning. New Yok: Penguin Group, pp. 61-73.
11. Krok, D. (2015). The role of meaning in life within the relations of religious coping and psychological well-being. Journal of Religion and Health, 54(6): 2292-2308.

12. Marco JH, Guillén V and Botella C. (2017). The buffer role of meaning in life in hopelessness in women with borderline personality disorders. Psychiatry research, 247: 120-124.

13. Martin RA, MacKinnon S, Johnson $\mathrm{J}$ and Rohsenow DJ. (2011). Purpose in life predicts treatment outcome among adult cocaine abusers in treatment. Journal of Substance Abuse Treatment, 40(2): 183-188.

14. Mc Andrew S and Warne T. (2014). Hearing the voices of young people who self-harm: Implications for service providers. International journal of mental health nursing, 23(6): 570-579.

15. Mohabbat-Bahar S, Golzari M, Moradi-Joo M and Akbari ME. (2014). Efficacy of group logotherapy on decreasing anxiety in women with breast cancer. Iranian journal of cancer prevention, 7(3): 165.

16. Park CL. (2010). Making sense of the meaning literature: an integrative review of meaning making and its effects on adjustment to stressful life events. Psychological bulletin, 136(2): 257.

17. Park CL. (2017). Distinctions to promote an integrated perspective on meaning: Global meaning and meaning-making processes. Journal of Constructivist Psychology, 30(1): 14-19.

18. Purdy J. (2013). Chronic physical illness: a psychophysiological approach for chronic physical illness. The Yale journal of biology and medicine, 86(1): 15.

19. Sadock BJ, Sadock VA, Ruiz P. (2015). Psychological Factors Affecting Other Medical Conditions. Synopsis of Psychiatry. Philadelphia: Wolters Kluwer, pp. 477-489.

20. Saeed AA, Bahnassy AA, Al-Hamdan NA, Almudhaibery FS and Alyahya AZ. (2016). Perceived stress and associated factors among medical students. Journal of family \& community medicine, 23(3): 166

21. Saleem S and Saleem T. (2017). Role of religiosity in psychological well-being among medical and non-medical students. Journal of religion and health, 56(4), pp.1180-1190.

22. Shand LK, Cowlishaw,S, Brooker JE, Burney $S$ and Ricciardelli LA. (2015). Correlates of post-traumatic stress symptoms and growth in cancer patients: A systematic review and meta-analysis. Psycho-Oncology, 24(6): 624-634.

23. Sousa N. (2016). The dynamics of the stress neuromatrix. Molecular psychiatry, 21(3): 302.

24. Steger MF and Kashdan TB (2013). The unbearable lightness of meaning: Well-being and unstable meaning in life. The Journal of Positive Psychology, 8(2): 103-115.

25. Steger MF, Mann JR, Michels P and Cooper TC. (2009) Meaning in life, anxiety, depression, and general health among smoking cessation patients. Journal of Psychosomatic Research, 67(4): 353-358.

26. Subramaniyan R, Veliah G. and Gopichandran V. (2014). Purpose in life and its association with stress among persons living in a semi-urban area of Tamil Nadu. Journal of postgraduate medicine, 60(4): 377.

27. Theoharides TC, Angelidou A, Alysandratos KD, Zhang B, Asadi S and et al. (2012) Mast cell activation and autism. Biochimica et Biophysica Acta (BBA)-Molecular Basis of Disease, 1822(1): 34-41. 
28. Yanga L, Zhaob Y, Wanga Y, Liua L, Lia XZB, et al. (2015). The Effects of Psychological Stress on Depression. Current Neuropharmacology, 13: 494-504.

29. Yau YH, and Potenza MN, (2013). Stress and eating behaviors. Minerva Endocrinologica, 38(3): 255-267.
30. Wilchek-Aviad Y and Malka M, (2016). Religiosity, meaning in life and suicidal tendency among Jews. Journal of religion and health, 55(2): 480-494

31. Kim, J., \& Seo, B. S., (2013). How to calculate sample size and why. Clinics in orthopedic surgery, 5(3), 235-242. 